\title{
Research on mergers and acquisitions of the international hotel group Take Marriott M \& A Starwood as an example
}

\author{
Junfeng Wang
}

Dalian Institute of Science and Technology, Lvshun, Dalian,Liaoning,China

345106969@qq.com

\begin{abstract}
Keywords: International hotel group, Mergers and Acquisitions, Rebranding,Brand integration
\end{abstract}
\begin{abstract}
The global hotel industry has begun to expand with mergers and acquisitions as its main form since the 20th century. M\&A has become an extremely important way for international hotel groups to expand their scale and increase their competitiveness. This document analyzes the main motivations for the acquisition of Starwood by Marriott, discusses the challenges and difficulties faced by Marriott and Starwood in the process of rebranding and brand integration, and proposes future rebranding and brand integration ideas.
\end{abstract}

\section{国际酒店集团并购研究 以万豪并购喜达屋为例 \\ 王俊峰 \\ 大连科技学院管理工程学院, 旅顺, 大连, 辽宁, 中国 345106969@qq.com}

关键词：国际酒店集团；并购；品牌重塑；品牌整合

中文摘要. 自 20 世纪以来, 全球酒店业开始了以并购为主要形式的扩张, 并购已成为国际酒 店集团扩大规模, 提升竞争力极为重要的途径。本文通过分析万豪并购喜达屋的主要动因, 探讨万豪与喜达屋品牌重塑和整合面临的挑战和困难，提出未来的品牌重塑和整合思路。

\section{1. 引言}

近几年，国内和国外酒店业并购风起云涌，线上线下联动成风，全球酒店业的并购事件 不管是并购金额还是并购次数, 都远超往年, 其中最为轰动的当属万豪成功并购喜达屋。此 次并购是名副其实的 “强强联合”，使万豪一跃成为全球最大的酒店集团。而并购后万豪如何 “消化” 喜达屋, 达到良好融合, 则是业内人士、学者、客户等群体共为关注和讨论的话题。

\section{2. 万豪并购喜达屋的主要动因剖析}

\section{1 快速扩张规模优势}

万豪并购喜达屋这一重大事件也充分说明了酒店行业的商业模式正面临着巨大压力, 在 全球经济不景气及行业发展缓慢等多种因素共同促使下, 两家酒店集团需要通过合并, 降低 成本, 变得更强大, 来抵御竞争对手。公开数据显示, 双方合并之后, 万豪可以节省 2 亿美元 
运营成本, 更重要的是万豪成为全球第一大酒店集团, 其酒店品牌数量、酒店数量以及客房 数量一跃成为全球第一。

\section{2 拓展海外市场, 填补市场空缺}

据万豪公布的 2014 年财报，万豪仅有 $26 \%$ 的客房国际市场份额。相比于希尔顿、喜达屋 等品牌, 其在海外市场的客房数量占比上垫底。而喜达屋则显得更为 “外向”，其在国际市场 现有、在建客房数量的市场额度分别为 $57 \%$ 和 $61 \%$, 这与万豪形成了鲜明对比。随着并购的完 成, 万豪在亚洲的市场规模将扩大一倍以上, 使万豪能够以最便捷的途径卡位海外市场, 弥 补自身短板（周芗，2016）。

\section{3 应对在线旅游服务的严重威胁}

喜达屋和万豪合并后将成为全球最大的酒店集团，提高不断被压缩的利润空间和议价能 力, 能够有效防范 Airbnb、OTA 的袭扰, 在未来为了可以努力争取与 OTA 合作中的话语权, 应对 Airbnb 这类强劲竞争者, 掌握市场的主动权。万豪通过并购, 可以实现自己的酒店+互 联网，而不是被动的受制于 OTA 的互联网+酒店（王强，2016）。

\section{4 丰富客户群体，促进品牌谱系的健全化}

合并后，万豪就能够快速拥有了吸引年轻一代客户群体的酒店品牌，在巩固自身在奢华 和高端领域的实力的基础上, 更是通过并购喜达屋来吸引年轻消费群体, 有效地丰富客户群 体, 填补和加强市场空缺。除了在奢华酒店等共同市场扩展了品牌阵容外, 此次并购更是结 合了万豪在豪华、度假、会议及精选服务酒店领域以及喜达屋在生活时尚酒店品牌及国际网 络的优势, 可为客人、酒店业主及特许经营合作伙伴、会议筹备商等带来更为全面的品牌阵 容（叶碧华，2015)。

\section{3. 万豪并购整合喜达屋面临的主要问题}

\section{1 品牌重塑难题}

作为国际知名酒店集团的万豪和喜达屋品牌, 合并后, 酒店的名称该如何改变？实施单 一品牌策略的公司希望用 “同一张脸” 与顾客熟识，使顾客在记忆中保持美好、鲜明、独特 的品牌联系（Keller，1993)。但带来的问题，如目标品牌与企业整体形象不符、难以保持品 牌传播连续性和统一性、品牌延伸之后的问题等, 或许会从消费者头脑中抹去原有品牌所激 发的积极形象 (Muzellec, 2005)，公司名称的更换会造成员工和顾客的疏离、降低品牌的亲 切感和导致顾客的混淆 (Edmonson, 2002) 等, 这也可能是并购后各自品牌还是依然独立运 营的原因和优势所在。同时，联姻后的万豪国际与喜达屋酒店将拥有 5500 家酒店，旗下的酒 店品牌总数达到了 30 个, 两家酒店目前品牌体系的重合度较高, 同质的酒店品牌定位相似, 消费群体重叠很高, 这些都有可能造成消费群体重合。双方合体后或将面临同一城市形成自 相竞争的状况，必须合理地区品牌分布以解决同质品牌的相互竞争问题。

\section{2 双方客户系统整合问题}

几乎每家大型连锁酒店都有常客奖励制度, 其中喜达屋优先客户计划在业内尤为突出, 十分受客户青睐。如果合并后, 原喜达屋的 SPG 计划不再实施或缩水, 势必会导致许多原喜 达屋忠实客户的离开。事实上, 对于喜达屋旗下各品牌酒店的常旅客来说, 喜达屋被万豪收 购, 就像是自己的 “母亲” 嫁给了一个自己并不喜欢的 “父亲”, 并不情愿。所以一旦处理不 好这种关系, “孩子出走” 的概率就会很大。

\section{3 管理方式与企业文化整合问题}

尽管喜达屋集团和万豪集团都有着非常丰富的酒店管理以及酒店并购的经验，但是正因 
为双方力量都是 “大牌”, 反而在许多问题, 如企业文化〉执行标准等方面难以融合, 这也是 很多企业并购的通病。有业内人士指出, 并购后的双方管理方式及文化必然需要融合, 这样 才能够更好的发挥协同效应，使得规模化、连锁化发挥真正的效应（周人果，2016)。但万豪 改如何 “消化” 喜达屋, 则是一个需要严肃思考的问题。同时, 随着整合的深入, 组织部门 变革、人员变动和整合也不可避免, 如何能够较为顺利地实现两个品牌的人才整合, 避免人 才的流失和管理波动, 实现两个品牌的人才融合, 产生更大合力, 这也是管理团队和业内人 士、员工当事人等多方面共同关心的问题。

\section{4. 万豪并购喜达屋的品牌重塑与整合思路探讨}

\section{1 审慎有序品牌重塑, 充分发挥品牌优势}

\section{1 .1 保持各自品牌的独立性}

万豪与喜达屋，同为国际酒店行业的豪华品牌，有着深厚的品牌文化沉淀、众多忠诚的 客户。从短期看, 在较长的一段时期内, 万豪与喜达屋旗下的各品牌保留原来的品牌名称、 标识等是明智之选, 无论是在品牌表面的名称等, 还是在各方面实际感受到的服务品质等, 不能让喜达屋的忠实客户觉察到被万豪 “侵袭” 的不良感受, 让忠实客户相信喜达屋还是原 来那个喜达屋, 依然还是那么优秀; 从长期看, 万豪的优秀基因、烙印融入喜达屋各品牌是 一个渐进的过程。

4.1.2 品牌整体重新定位分类与品牌精简重组

由于万豪国际与喜达屋旗下酒店品牌在客户中已有相当高知名度和固定形象，有业内人 士担心合并后或将产生品牌认知混淆 (孙伶, 许望, 2016), 显然, 万豪并购喜达屋后, 其规 模已经足够大, 如果不能很好地 “消化” 喜达屋, 很可能导致一些品牌会逐渐消亡。因此, 未来万豪会对旗下的 30 个酒店品牌做出更具有特色的再定位, 提升品牌服务的标准。从短期 来看, 万豪不应对现有的两家酒店品牌进行较大的变动。但从长期来看, 为了更有效的品牌 管理, 避免品牌和网点重合, 品牌精简和优化可能是必然的趋势。

\section{2 合理处理礼赏计划和SPG计划之间的关系}

会员计划方面, 虽然从两家的数量上看, 万豪具有压倒性优势, 但是在会员计划的受欢 迎程度上看, 喜达屋则明显更胜一筹（秋薇，2016）。合并后, 两家集团合并后的常旅客奖 励计划可能是, 在短期内, 礼尚计划与 SPG 继续独立保持运作, 双方可以针对某级别的精英 会员设置一些共享的互惠政策, 或实现三个常客计划的账户联合, 实现三个计划之间的积分 兄换, 从而享受三个常客计划下更多更好的服务。未来, 礼赏计划将逐渐吸取采纳一些 SPG 的优惠项目，譬如套房升级优惠等，逐步实现两者的融合。

\section{3 有效融合企业文化}

在企业文化方面，万豪集团与喜达屋集团都各有其优势。万豪的 20 个管理理念，服务社 会的精神、微笑服务, 喜达屋的明星服务标准、十诫以及喜达屋关爱, 这些企业文化都有相 似的地方。但是如果在合并后, 企业文化依然保持其各有的标准, 这对拥有 30 个品牌的万豪 集团发展来说显然是不利的, 可能会造成管理的混乱, 比如员工培训、员工服务都可能出现 偏差。所以, 万豪一定要充分整合, 升级它的一系列标准, 推广它的企业文化, 在推广过程 中还要考虑推广难度, 还需要形成一套新的培训机制, 否则在企业文化这方面无法深入贯彻 到员工心中。

\section{5. 结束语}

万豪并购喜达屋, 成就了更强大的万豪, 有效加强了其应对线上和线下威胁的能力。但 
两者的有效整合还任重道远，在品牌重塑、企业文化、客户系统融合等诸多方面还有许多工 作要做。同时, 本案例并购整合过程中的品牌、人员、会员计划、企业文化等方面整合和重 塑思路、经验等也值得其他酒店管理集团，尤其是中国的酒店管理集团学习和借鉴。

\section{References}

[1] Aaron Smith, Marriott and Starwood merge: World's biggest hotel company, CNN, November $16,2015$.

[2] David Taylor, Marriott+Starwood : The result of this marriage is happiness or worry? , New Marketing, vol.1, pp. 8-8, 2016.

[3] Edmonson K, A case of mistaken identity: Governance and the screen sounds age, Journal of the Australian Society of Archivists, 2002,30 (1) :30-46.

[4] Keller K L, Conceptualizing, measuring, and managing customer - based brand equity, Journal of Marketing, 1993, 57 (1):1-22.

[5] MARRIOTT INTERNATIONAL AND STARWOOD HOTELS \& RESORTS WORLDWIDE SIGN AMENDED MERGER AGREEMENT, www.marriott.com, March 21,2016.

[6] Muzellec L, what's in a name change? Re-Joining corporate names to create corporate brands, Corporate Reputation Review, 2005, 8 (4): :44-48.

[7] Wei Qiu, Marriott Group CEO Proclaims Starwood Member: Hey, Don't Want Your Life, Tripvivid Travel, 2016.2.29.

[8] Wei Sun, Wang $\mathrm{Xu}$, International hotel group mergers and acquisitions still hot, local brands overseas rapid expansion, 21st Century Business Herald, 2016.3.14 .

[9] Bihua Ye, Marriott International's 12.2 billion U.S. whale swallowed Starwood, Chinese and foreign hotels accelerating competition, 21st Century Business Herald, 2015.11.18.

[10]Renguo Zhou, The hotel industry lifted the wave of mergers and acquisitions, Nanfang Daily, 2016.5.

[11]Ying Zhou, Marriott: M\&A is King, New Wealth, 2016, (2) :38-45 